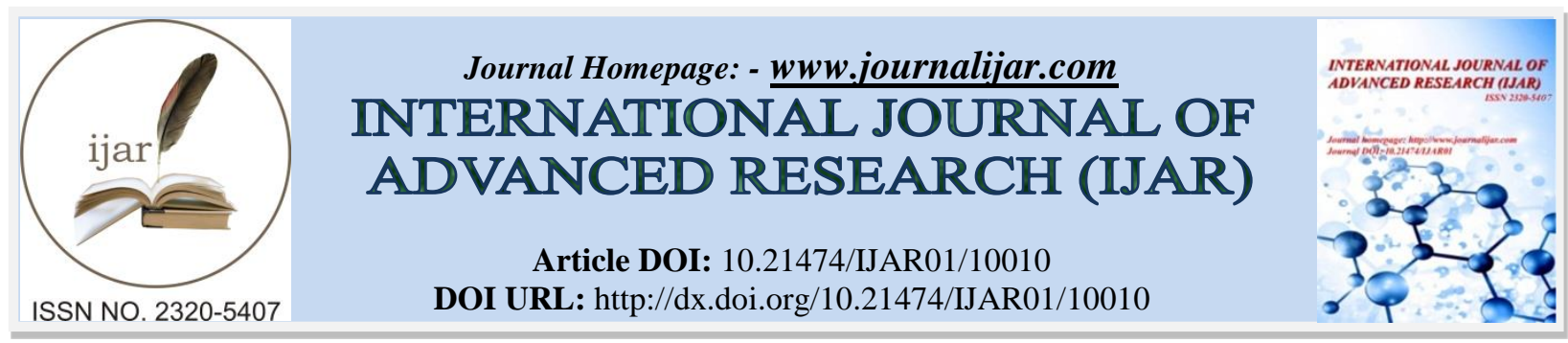

RESEARCH ARTICLE

\title{
DIAGNOSIS OF PULMONARY TUBERCULOSIS USING CHEST X-RAYS.
}

Prakanshu Srivastava ${ }^{1}$,Vivek Kumar Sahu ${ }^{1}$ Sachin Singh $^{1}$, Vibhav Awasthi ${ }^{1}$ and Isha Sehgal ${ }^{2}$.

1. Student, PSIT College of Engineering, Dept. of Information Technology Kanpur Uttar Pradesh, India.

2. Assistant Prof., PSIT College of Engineering, Dept. of Information Technology Kanpur Uttar Pradesh, India.

\section{Manuscript Info}

Manuscript History

Received: 08 September 2019

Final Accepted: 10 October 2019

Published: November 2019

Key words:-

Tuberculosis, Chest X-Rays(CXR),

Lungs, Pulmonary.

\section{Abstract}

Tuberculosis is a major drawback and speedily unfold disease in all over the world. Correct diagnosis is the key to controlling the disease. Ancient ways like tuberculin skin test (TST) results that take longer to find T.B. This paper presents an automatic approach to find T.B. using chest X-Rays. T.B's first and foremost target is to attack the human respiratory system and to disturb its traditional functioning by affecting most specific a part of the respiratory system i.e. lungs. It becomes tough to breathe properly with the affected lungs and if not timely treated, the severity of the disease can increase and it can result in death.

Copy Right, IJAR, 2019,. All rights reserved.

\section{Introduction:-}

With each passing day, almost everything is being automatic with the assistance of machines that may think on their own. Health care is one of the basic aspects of human life. About two billion individuals are infected with tuberculosis worldwide[1]. Pulmonary tuberculosis (PTB) is a chronic disease common among crowded and poorly ventilated areas.

1. TB is a chronic or acute infection which is caused by mycobacterium. It causes respiratory organ infiltrates i.e. the development of granulomas along with caseation, cavitation or fibrosis. Tuberculosis is an infectious disease that primarily affects the respiratory organ parenchyma[2].

2. It conjointly may be transmitted to alternative parts of the body, together with the membrane, kidneys, bones, and lymph nodes.

3. The primary infectious agent, M. tuberculosis, is an acid-fast aerobic rod that grows slowly and is sensitive to heat and UV light[3].

Tuberculosis (TB) is an old disease - studies of human skeletons show that it has affected humans for thousands of years. In the late 1800s, cause-of-death data from national vital registration systems show that TB was one of the leading causes of death in some European countries. With social and economic development - such as improvements in incomes, housing and nutrition - numbers of TB cases and deaths started to decline in western Europe, North America and some other parts of the world around the turn of the 20th century, albeit slowly (1-2\% per year). From the 1940s, the discovery, development and use of effective drug treatments substantially accelerated these trends, with national case rates (per 100000 population) falling by up to $10 \%$ per year and mortality rates falling even faster. In countries that have experienced such reductions in disease burden, and now have only around 10 or fewer cases and less than 1 death per 100000 population per year, TB is often considered a disease of the past[4]. 


\section{Background \\ Chest X-ray for detecting TB}

Chest X-ray (CXR) is a rapid imaging technique that allows lung abnormalities to be identified. CXR is used to diagnose conditions of the thoracic cavity, including the airways, ribs,lungs, heart and diaphragm[5].

CXR has historically been one of the primary tools for detecting tuberculosis (TB), especially pulmonary TB. CXR has high sensitivity for pulmonary TB and thus is a valuable tool to identify TB as a differential diagnosis for patients, especially when the X-ray is read to identify any abnormality that is consistent with TB. However, CXR has poor specificity; although some CXR abnormalities are rather specific for pulmonary TB (for example, cavities), many CXR abnormalities that are consistent with pulmonary TB are seen also in several other lung pathologies and, therefore, are indicative not only of TB but also of other pathologies. Moreover, there is significant intra- and interobserver variation in the reading of CXRs. Relying only on CXR for TB diagnosis leads to overdiagnosis, as well as underdiagnosis . Rigorous efforts should always be made to base a TB diagnosis on bacteriological confirmation (sputum-smear microscopy, culture or a molecular test). WHO classifies TB diagnosis into bacteriologically confirmed TB[6], if it is based on bacteriological confirmation, or clinically diagnosed TB, if it is based on clinical assessment including CXR, but is not confirmed by bacteriological examination.

\section{Image Processing:}

Image process is a methodology to perform some operations on a picture, so as to induce an increased image[7] or to extract some helpful data from it. it's a sort of signal process during which input is a picture and output could also be image or characteristics/features related to that image. Nowadays, image process is among quickly growing technologies. It forms core analysis space among engineering and engineering science disciplines too.

Image process primarily includes the subsequent 3 steps:

1. Importing the image via image acquisition tools

2. Analysing and manipulating the image

3. Output during which result will be altered image or report that's supported image analysis.

\section{Methodology:-}

$\mathrm{X}$-ray imaging is based on the fact that tissue will absorb photons from an X-ray beam in relation to the electron density of the tissue. This means that bones absorb more photons than lean tissue does. The number of photons passing through the body of interest will then be detected either by film or now by image detectors that convert the body's direct attenuation of the photons into digital images. The resulting images are a two-dimensional projection of a three-dimensional structure[8]. A disadvantage of X-ray-based techniques is the ionizing radiation, which limits the usefulness of these techniques for longitudinal studies.

\section{Image Enhancement:}

Image enhancement refers to sharpening of image features such as edge boundaries or contrast to make graphic display more useful for display and analysis. Image enhancement includes grey level and sharpening, filtering, noise removal and so on. 

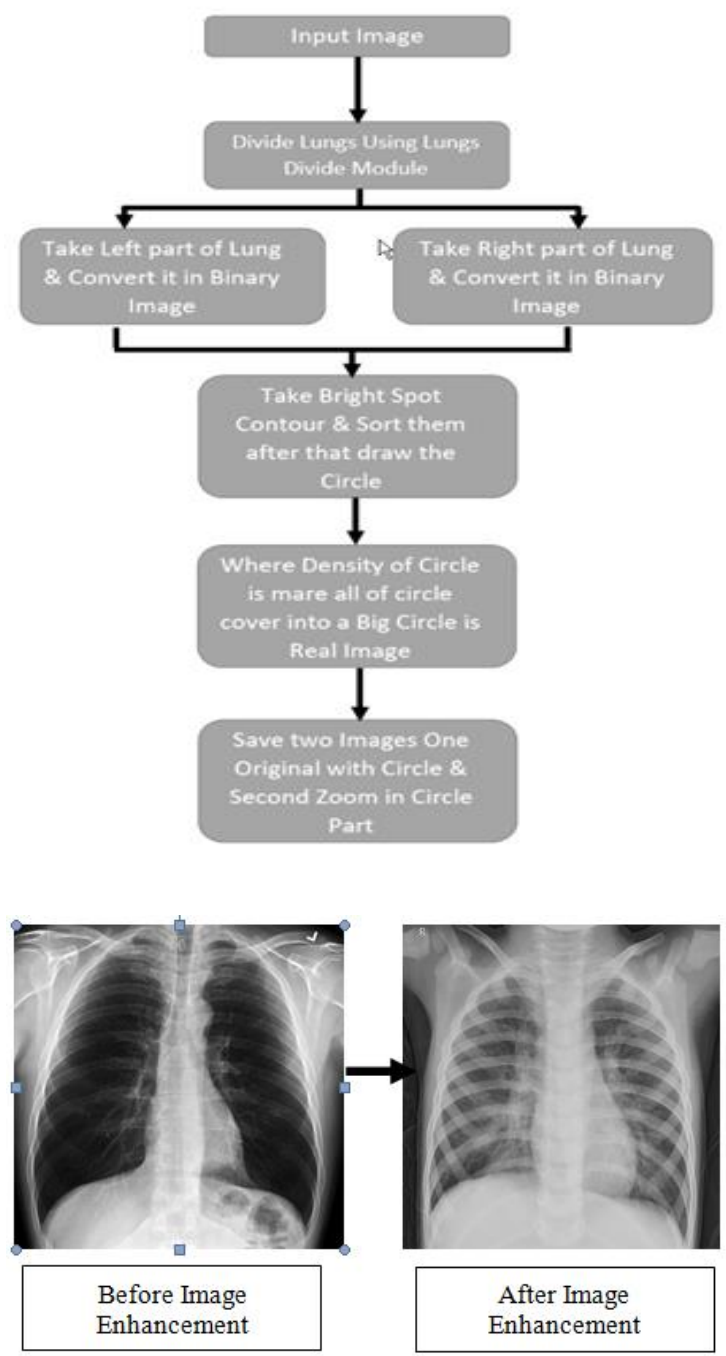

Dividing the Lungs into two parts using Lung Finder Library:

Image enhancement refers to sharpening of image features such as edge boundaries or contrast to make graphic display more useful for display and analysis. Image enhancement includes grey level and sharpening, filtering, noise removal and so on. The lung zones do not equate to the lung lobes. We used Lung Finder library for detecting Lungs on chest X-Ray images for further processing and divide[11] it into two parts(Left and Right).

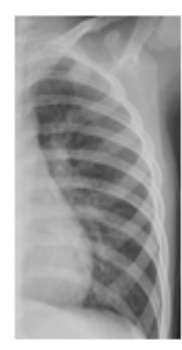

filtered.

$$
\begin{gathered}
\text { Divided } \\
\text { Part }
\end{gathered}
$$

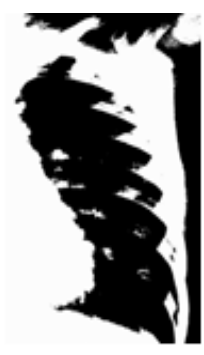

ROI Image 


\section{Creating the Binary Mask (ROI):}

This section describes how to create binary masks to define ROIs. However, any binary image can be used as a mask, provided that the binary image is the same size as the image being filtered.

\section{Filtering Image using Image Processing:}

Filters are mainly used to suppress either the high frequencies in the image, i.e. smoothing the image, or the low frequencies, i.e. enhancing or detecting edges in the image. Noise removal is easier in the spatial domain as compared to the frequency domain as the spatial domain noise removal requires very less processing time. The two types of filters are the linear and non-linear filters. The linear filters have the advantage of faster processing but the disadvantage of not preserving edges. Conversely the non-linear filters have the advantage of preserving edges and the disadvantage of slower processing.

\section{Make Contours:}

Before starting the image undergoes light blurring to eliminate spot noise. The best results were obtained using bilateral iteration algorithm. As a preliminary, a binary version of input image I bin is computed with a threshold evaluated with Otsu's method . In order to get a more consistent and integral result, morphological dilation with small disk radius can be applied to I bin. Draw small circles at all bright spot contours.

\section{Final Distorted Section:}

Mark the spot where the density of small circle is very high. Draw a bigger circle surrounding all the small circles at the high density area.

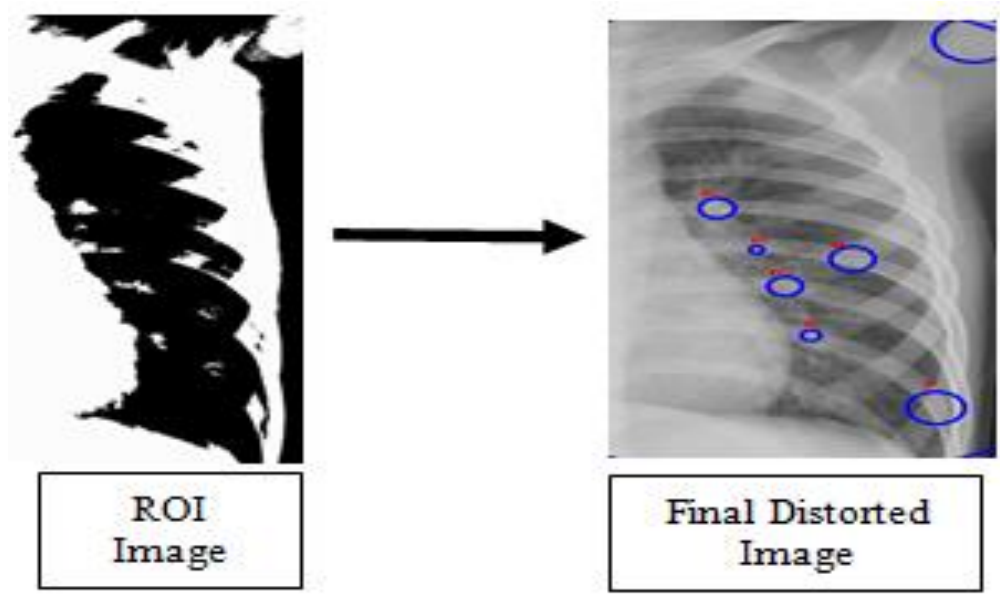

\section{Features}

Accuracy in results can be seen within very low time consumption along with less harm to body from the $\mathrm{x}$ rays as there is no need to get the $\mathrm{x}$ rays again and again for observation. Faster treatment can be given to the patient as the area affected and the level of infection can be determined easily.

\section{Conclusion And Future Works:-}

Conclusion-Posterior-anterior chest X-ray image view contains a lot of primary information about the disease. The cardiac size and that proportioning the diameter to the thoracic diameter is established as cardiothoracic ratio. That ratio is 0.5 or higher is careful to abnormal[9]. Examining a particular region of interest in an image, set by processing the information in the region provides a diagnosis of the region. That suspected region in chest X-ray require careful examination by doctor and can give alarm for particular cases which need urgent attention.[10] Chest $\mathrm{X}$-ray is an easy and economical screening test for any patient even in the rural setup, it can be considered as a groundwork evaluation to the size of the heart, however the advance imaging techniques stay additional correct[11]. It can be used in every hospitals to avoid the fatality of the disease at the initial stage. It provides facility of proper treatment at low cost to the patients who cannot afford the treatment. It can also be used for education purpose in medical colleges. 


\section{Acknowledgement:-}

The authors would like to thank the anonymous referees for their valuable comments that improved this paper greatly. A vote of thank to Ms. Isha Sehgal for her guidance and supervision to perform this research.

\section{References:-}

1. http://www.who.int/trade/distance_learning/gpgh/gp gh3/en/index4.html

2. https://doctorlib.info/physiology/pathophysiology/42.html

3. https://nurseslabs.com/pulmonary-tuberculosis/

4. World Health Organisation. The worldwide magnitude of protein-energy malnutrition: an overview from the World Health Organisation. Provisional guidelines for the diagnosis and classification of the EPI target diseases for primary health care, surveillance and special studies, EPI/GEN/83/4. Geneva: World Health Organisation, 1983.

5. Wai Yan Nyein Naing, Zaw Z. Htike, "Advances in automatic tuberculosis detection in chest X-ray images", signal \& image processing an international journal (sipij) vol.5, no.6, December 2014

6. V. Cleeff, L. E. Kivihya-Ndugga, H. Meme, J. A. Odhiambo,and P. R. Klatser, "The role and performance of chest Xray for the diagnosis of tuberculosis: A cost-effectiveness analysis in Nairobi, Kenya", 2005.

7. Joshua M. Leibstein and Andre L. Nel, "Detecting tuberculosis in chest radiographs using image processing techniques", School of Electrical and Electronic Engineering University of Johannesburg

8. H. Das and A. Nath, "An Efficient Detection of Tuberculosis from Chest X-rays," in International Journal of Advance Research in Computer Science and Management Studies, Vol. 3, Issue 5, p.p. 149-154, May 2015

9. CHEST RADIOGRAPHY IN TUBERCULOSIS DETECTION, WHO Report 2018.

10. Betsy Antony, Nizar Banu P K, Lung Tuberculosis Detection Using X-Ray Image, nternational Journal of Applied Engineering Research ISSN 0973-4562 Volume 12, Number 24 (2017) pp. 15196-15201.

11. https://pypi.org/project/lungs-finder/ 